\title{
A Sustainable System for Improving Energy Performances Applicable to the Existing Collective Housing
}

\author{
Jo, Mu-Jin* $\cdot$ Han, Seung-Hoon ${ }^{* *}$ \\ *EAN Technology, South Korea (iloveyureka@naver.com) \\ ** Corresponding Author, School of Architecture, Chonnam National University, South Korea (hshoon@jnu.ac.kr)
}

\section{A B STR A C T}

\begin{abstract}
Purpose: Currently, there are many success stories coming out various energy-saving / production or eco-friendly buildings. However, these case and method didn't consider of application with existing housing and high-rise housings. In the case of Europe, the North America is gradually grew and settle through the voluntary, small, private development. But this method and system are not fit for the majority of developing countries including South Korea. Method: In this situation, this paper analyse, first arranged previous research and case study, second divided factors and re-organized factors, third analysed plan and elevation of apartment and selected main plan type and elevation type of apartment, finally analysed method of application with existing buildings and high-rise buildings by test and simulation. Result: In sum, this research finally analyzed the change of electricity and fuel consumption according to the change of insulation standard. This study has been expected to serve as a bridge of the energy housing system development and suggest new method applied to the existing housing and building.
\end{abstract}

\begin{tabular}{l} 
K E Y W O R D \\
\hline 에너지시스템 \\
기축건축물 \\
지속가능 환경조절 \\
건축에너지성능 \\
실내환경품질 \\
Energy Production System \\
Existing Housing and Buildings \\
Sustainable Energy Platform \\
Building Energy Performances \\
Environmental Quality \\
A C C E P TA N C E IN F O \\
\hline Received Sep 24, 2017 \\
Final revision received OCT 18, 2017 \\
Accepted OCT 23, 2017
\end{tabular}

\section{에너지시스템}

기축건축물

Energy Production System

isting Housing and Buildings

Sustainable Energy Platform

Environmental Quality

Received Sep 24, 2017

Accepted OCT 23, 2017

\section{INTRODUCTION}

The total consumption of households in Korea accounts for more than $10.5 \%$ of total domestic energy consumption. Therefore, energy saving efforts has been variously researched and developed in the residential area. As part of energy saving efforts, it is also important to reduce energy consumption of existing buildings and to use them efficiently. Government also was enacted the "Greenhouse Gas Emission Trading Act" on January 1, 2015 to reduce greenhouse gas emissions, purchase emission credits, and established a green building certification system (G-SEED) for buildings that contributed to energy conservation and environmental pollution reduction based on certification standards.

The current trend of domestic research on apartment remodeling can be seen before and after the change of the characteristics of apartment remodeling to the improvement of energy performance. Studies of the apartment remodeling before the change of the energy performance improvement are mainly focused on the status, situation of remodeling and policy improvement for aged apartment. J. Bae (2004) researched to the status of remodeling of apartment and institutional improvement policy, and E. Kim (2010) researched on growth plans and improvement prospects.

pISSN 2288-968X, eISSN 2288-9698 http://dx.doi.org/10.12813/kieae.2017.17.5.025
After the change, many studies focused on the remodeling plans for improving the energy performance of existing houses such as green remodeling. The Ministry of Land (2013) proposed a remodeling plan for revitalizing the energy performance of existing buildings.

Also, the government aims to provide million of green homes by serve some of the installation fee when installing new and renewable energy facilities in homes until 2020. However, the above policy and various cases of national and international are limited to only new construction, and even there is a limit to researching actual contents by building them for exhibition hall or experimental purposes.

The purpose of this research is to analyze the difference of energy performances according to the change of elevation shape by remodeling apartment houses using simulation. For this research, basic methods and advanced references by case studies for energy performance towards national and international buildings. And this research also reorganized factors of zero-energy house system that were limited to new and single houses to be applicable to the existing apartments, and suggested a system that can improve the energy performance on those housing types.

Through this process, this research simulated the energy performance by modeling 3D representative form of apartment unit in the 1970s and 1990s for comparative evaluation of reorganized 
factors and the suggested system. Also this study compared the energy performance before and after the remodeling of the existing apartment by the simulation using an energy analysis program associate to Revit, Green Building Studio, EnergyPlus and Ecotect. And this research finally aims to propose one of the best solutions and/or a method for remodeling houses by comparison of the result values.

\section{PRELIMINARY RESEARCH}

\subsection{Analysis of Basic Statistics}

1980 status of housing

1990 status of housing
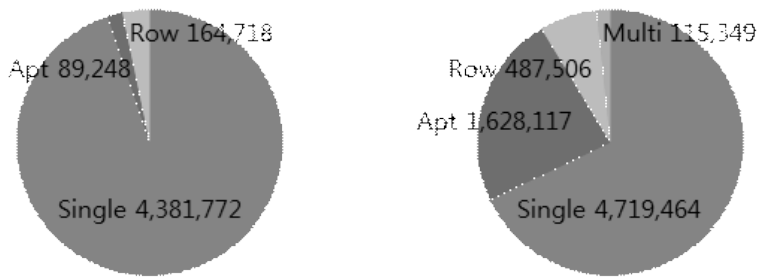

2000 Status of housing

\section{0 status of housing}
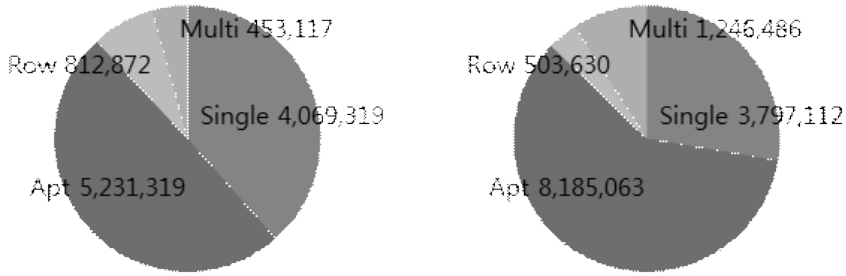

Fig. 1. Yearly Housing Situation in South Korea

Table 1. Increase / Decrease in Number of Single-family houses and apartments by year

\begin{tabular}{c|c|c|c|c}
\hline Year & $\begin{array}{c}\text { Number of } \\
\text { Apartments }\end{array}$ & $\begin{array}{c}\text { Increase } \\
\text { /Decrease }\end{array}$ & $\begin{array}{c}\text { Number of } \\
\text { Single houses }\end{array}$ & $\begin{array}{c}\text { Increase } \\
\text { /Decrease }\end{array}$ \\
\hline 1975 & 89,248 & & $4,381,772$ & \\
\hline 1980 & 373,710 & $+284,462$ & $4,652,127$ & $+270,355$ \\
\hline 1985 & 821,606 & $+447,896$ & $4,719,464$ & $+67,337$ \\
\hline 1990 & $1,628,117$ & $+806,511$ & $4,726,933$ & $+7,469$ \\
\hline 1995 & $3,454,508$ & $+1,826,391$ & $4,337,105$ & $-389,828$ \\
\hline 2000 & $5,231,319$ & $+1,776,811$ & $4,069,463$ & $-267,642$ \\
\hline 2005 & $6,626,957$ & $+1,395,638$ & $3,984,954$ & $-84,509$ \\
\hline 2010 & $8,185,063$ & $+1,558,106$ & $3,797,112$ & $-187,842$ \\
\hline
\end{tabular}

Prior to the study, most of the energy efficient residential systems (Zero Energy House System, Passive House System, Green House System) serve to newly constructed and single- family houses. On the contrary, the current Korea housing situation shows that new construction is steadily decreasing. The number of apartments still shows a steadily increase, but the number of single-family houses is on the decline. Also, new construction of domestic housing is decreasing, and its construction has exploded in the 1970s and experienced rapid quantitative growth until the 1990s. However, it started to decline gradually in the 2000s, and now is sharply decreasing as shown on Fig. 1.

\subsection{Definitions and Glossary of Term}

1) Definition of Apartment House

An apartment is a residential house that can be used as an independent residence for each household. at the same time, sharing all or part of the wall, corridors, stair, and other facilities of on building. The most type of apartment house is apartment, and there are also tenement houses and multi-family houses.

- Apartment

A house with five or more floors

- Town house

A house with a total floor area of more than $660 \mathrm{~m}^{2}$ and a floor number of 4 or less

- Tenement

A house with a total floor area of $660 \mathrm{~m}^{2}$ or less and a floor number of 4 or less

- Dormitory

A house used for students or employees such as schools or factories. it can do joint catering but does not have the form of independent residence.

\section{2) Definition of Remodeling}

In general, remodeling is defined as a construction activity that covers the maintenance, repair of existing buiildings, as opposed to new construction. As the construction industry, which has been focusing on quantitative growth, has pursued qualitative growth due to changes in the perception of users and society. Therefore, remodeling plays am important role in the currently construction industry and progresses for various purposes in structural, functional, economic and environmental aspects.

In addition, the early reconstruction of buildings that can be used for a long period of time causes national waste and causes construction costs and social costs. Therefore, remodeling is one of the solutions to solve this problem, and it is highly recommended from a policy aspects. 


\section{CLASSIFICATION AND SIMULATION}

\subsection{Classification and Reorganization of Factor}

First step of the experiment is re-organization to applied factors of smart energy house by previous studies and case studies. And applied factors are divided into the passive technology group, active technology group and renewable energy technology group. Then, these classified factors are re-organized with applicable to existing housing, apartment housing.

As a result, re-organized factors are as follows : Shading Device, Green Wall \& Roof, High Insulation, High Airtightness, High Efficiency Window, Natural Ventilation, Solar Energy Generation, and Wind Energy System. These factors have commonly features like easy to apply, fewer limit of apply and high efficiency energy saving.

\subsection{Type of Apartment House}

\section{1) Analysis Target}

With looking at the standards for remodeling in Korea, that is extended to building within $30 \%$ of the private housing area of each household when 15 years had passed after obtaining approval for use. Therefore, the analysis types of this paper are targeted and focused on apartment houses built by Korean National Housing Corporation from 1990s to 2000s that are older than 15 years. The period of 1980s and 1990s show quantitative growth centering on apartment complexes in Korea.

Also after 1993, the apartment complex business and plan continually focused on large-scale residential development projects in the surrounding areas of the metropolitan area, when two millions of houses were constructed completely. Therefore, the apartment complexes supplied at that time still occupy a large proportion and need to be analyzed as an object of immediate remodeling. And, the analysis setting focuses on apartment complexes built by Korea National Housing Corporation.

\section{2) Typification}

Table 2 and Table 3 show the result of the analysis of apartment complexes in the 1990s-2000s. Through these summarized tables, it turned out that changes of the housing type have led the modification of the heating system and configuration of the building components.
Table 2. 1990s Apartment Projects by Korea Housing Corporation

\begin{tabular}{|c|c|c|c|c|c|}
\hline & Region & \begin{tabular}{|c|} 
Name \\
of Apartment \\
Complex
\end{tabular} & $\operatorname{Area}\left(\mathrm{m}^{2}\right)$ & $\begin{array}{c}\text { Year } \\
\text { Completed }\end{array}$ & $\begin{array}{l}\text { Number of } \\
\text { household }\end{array}$ \\
\hline \multirow{3}{*}{1} & \multirow{3}{*}{ Panam-dong, Daejeon } & Jugong-2 & 64-105 & 1990.01 & 1130 \\
\hline & & Jugong-1 & $63-105$ & 1990.03 & 1016 \\
\hline & & Jugong-5 & $50-78$ & 1992.08 & 1436 \\
\hline \multirow{8}{*}{2} & \multirow{8}{*}{ Haan-dong, Gyunggi } & Jugong-10 & $60-105$ & 1990.03 & 2032 \\
\hline & & Jugong-6 & $52-71$ & 1990.03 & 1260 \\
\hline & & Jugong-7 & $63-105$ & 1990.03 & 1342 \\
\hline & & Jugong-8 & $63-105$ & 1990.03 & 1680 \\
\hline & & Jugong-5 & $63-105$ & 1990.05 & 2176 \\
\hline & & Jugong-11 & $52-69$ & 1990.06 & 1080 \\
\hline & & Jugong-9 & $63-105$ & 1990.09 & 1818 \\
\hline & & Jugong-12 & $59-104$ & 1990.11 & 2392 \\
\hline 3 & $\begin{array}{l}\text { Munheung-dong, } \\
\text { Gwangju }\end{array}$ & Woosan-2 & $63-82$ & 1990.06 & 1138 \\
\hline \multirow{2}{*}{4} & \multirow{2}{*}{ Sunbu-dong, Gyunggi } & Jugong-11 & $49-58$ & 1990.08 & 1190 \\
\hline & & Jugong-15 & $49-68$ & 1993.04 & 1210 \\
\hline 5 & Mansu-dong, Inchoen & Jugong-8 & $50-58$ & 1990.12 & 1440 \\
\hline 6 & Gyesan-dong, Inchoen & Jugong & $52-72$ & 1991.04 & 1140 \\
\hline \multirow{2}{*}{7} & \multirow{2}{*}{ Chang-dong, Seoul } & Jugong-3 & $58-105$ & 1991.05 & 2856 \\
\hline & & Jugong-4 & $52-72$ & 1991.11 & 1710 \\
\hline 8 & Bun-dong, Seoul & Jugong-1 & $58-105$ & 1991.05 & 1430 \\
\hline$\vdots$ & $\vdots$ & $\vdots$ & $\vdots$ & $\vdots$ & $\vdots$ \\
\hline 85 & $\begin{array}{c}\text { Bunpyung-dong, } \\
\text { Chungju }\end{array}$ & Jugong-4 & $56-81$ & 1999.07 & 1314 \\
\hline 86 & $\begin{array}{c}\text { Wonchun-dong, } \\
\text { Gyunggi }\end{array}$ & Jugong-2 & $70-106$ & 1999.09 & 1835 \\
\hline 87 & $\begin{array}{c}\text { Yeongtong-dong, } \\
\text { Gyunggi }\end{array}$ & Jugong-9 & $68-82$ & 1999.12 & 1265 \\
\hline 88 & Gyo-dong, Gangwon & $\begin{array}{l}\text { Gyodong } \\
\text { Jugong-1 }\end{array}$ & $65-110$ & 1999.12 & 1019 \\
\hline 89 & Damgam-dong, Busan & $\begin{array}{l}\text { Baeyang } \\
\text { Purenchae }\end{array}$ & $68-101$ & 2000.02 & 1116 \\
\hline \multirow{3}{*}{90} & \multirow{3}{*}{ Busan-dong, Gyunggi } & $\begin{array}{c}\text { Unam } \\
\text { Jugong-1 }\end{array}$ & $61-105$ & 2000.03 & 1755 \\
\hline & & $\begin{array}{c}\text { Unam } \\
\text { Jugong-2 }\end{array}$ & $102-102$ & 2000.11 & 1036 \\
\hline & & $\begin{array}{c}\text { Unam } \\
\text { Jugong-3 }\end{array}$ & $62-108$ & 2000.05 & 1651 \\
\hline 91 & Gojan-dong, Gyunggi & Neobill-6 & $92-103$ & 2000.05 & 1043 \\
\hline 92 & Jungbu-dong, Yangsan & $\begin{array}{l}\text { Yangsan } \\
\text { Jugong }\end{array}$ & $52-77$ & 2000.06 & 1248 \\
\hline 93 & Sinlim-dong, Seoul & $\begin{array}{c}\text { Samsungsan } \\
\text { Jugong-3 }\end{array}$ & $75-146$ & 2000.08 & 1482 \\
\hline 94 & $\begin{array}{l}\text { Dukjung-dong, } \\
\text { Gyunggi }\end{array}$ & $\begin{array}{l}\text { Bongwoo } \\
\text { Jugong-5 }\end{array}$ & $69-82$ & 2000.08 & 1732 \\
\hline 95 & Goam-domg, Gyunggi & $\begin{array}{c}\text { Juwon } \\
\text { Jugong-2 }\end{array}$ & $70-84$ & 2000.11 & 1935 \\
\hline
\end{tabular}


A Sustainable System for Improving Energy Performances Applicable to the Existing Collective Housing

Table 3. Analysis of Frequency by Type of Plan in 1990s

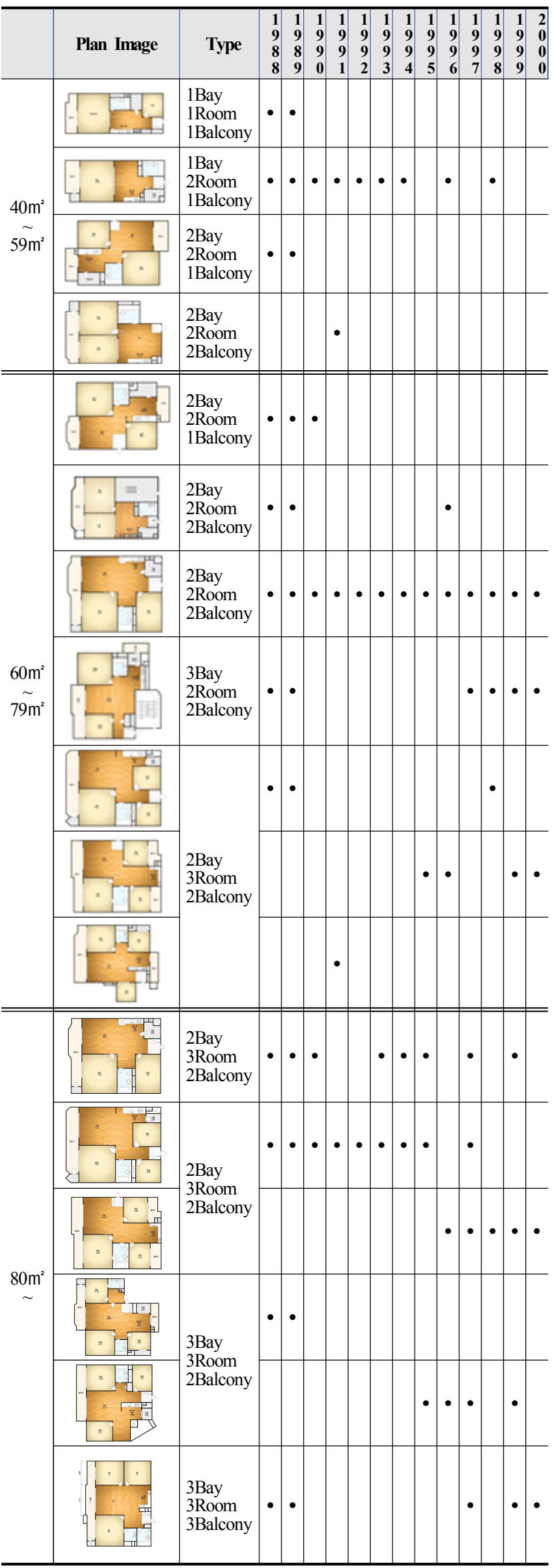

Table 4. Result of Type Analysis and Simulation Setting

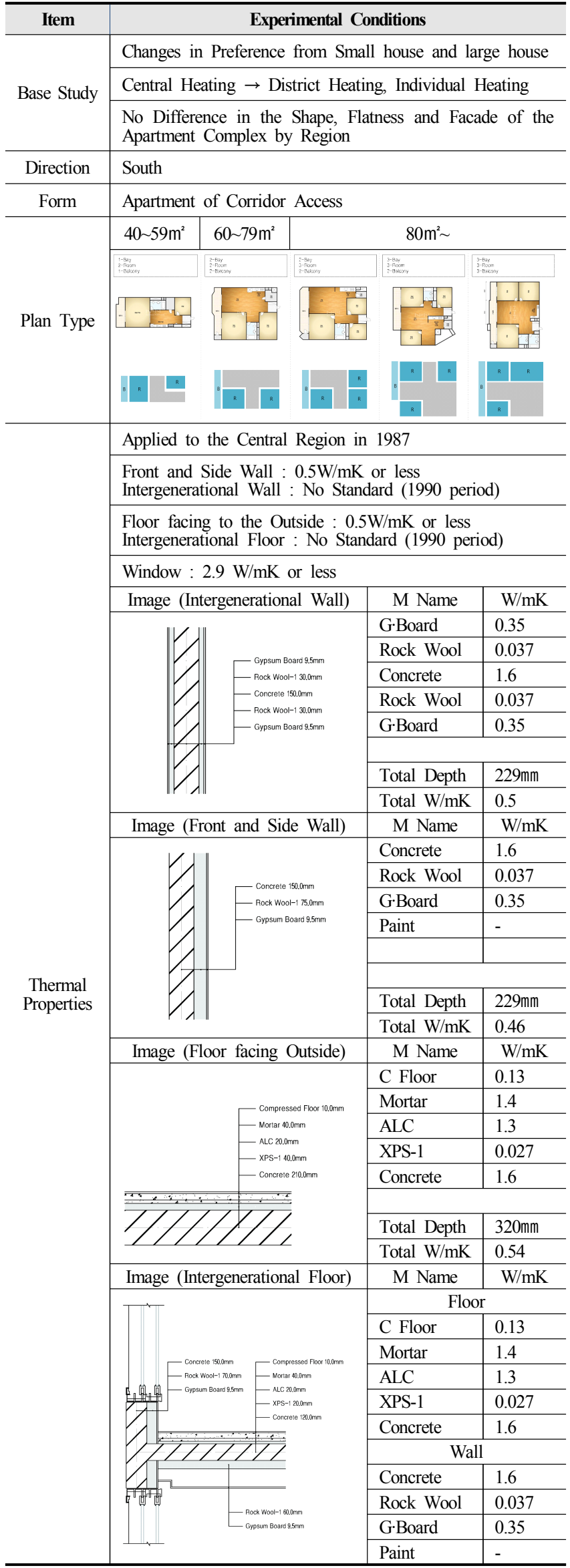




\subsection{Simulation}

Obviously, 3D models are constructed for reflecting thermal data and representing plan types in the process of classification. They are commonly set for the south orientation and a typical structure that is composed of corridors, RC walls, $210 \mathrm{~mm}$-thick floor slabs, and $150 \mathrm{~mm}$-thick outside and intergenerational walls.

This research classified the apartment houses between 1990s and 2000s in aspects of area and plan types, and then constructed 3D models for simulation. After reflecting common data to five types, this research tested performance and effect of energy-saving factors applicable to the existing apartment houses and analyzed changes of energy usage with Revit. As an ongoing issue, this study will have more tests on light, heat and energy performance by energy simulator like EnergyPlus.

1) Simulation Program

\section{- Revit}

Autodesk Revit is a BIM (Building Information Modeling) application for architectural experts such as designers, structural engineers, builders, MEP third vendors, contractors and so on. This software shows a capability to support designing a building in 3D using libraries. It also annotate the model with $2 \mathrm{D}$ design elements by accessing building information from the database. Revit is also used for construction in aspects of 4D navigation with tracking various stages through lifecycle of a building.

EnergyPlus \& Open Studio

EnergyPlus is a program developed by US Department of Energy (DOE) that integrates the merits of existing programs (DOE-2, BLAST). It is a precise simulation program that uses heat balance method. And this program is passible actual load calculation, modeling building mechanical system (boiler, chiller, cooling tower, fan, pipe, duct, etc.) and simulation the dynamic behavior of buildings and systems.

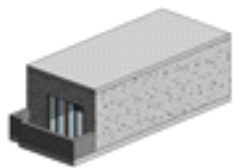

3D Model \#1 (1Bay, 2Room, 1Bal)

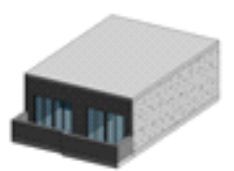

3D Model \#3 2Bay, 3Room 2Bal)

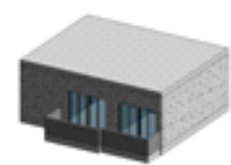

3D Model \#4

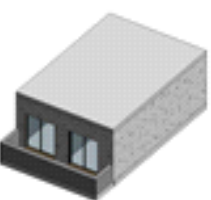

3D Model \#2 (2Bay, 2Room, 2Bal)

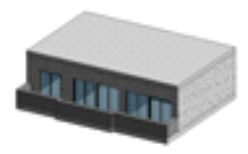

3D Model \#5 (3Bay, 3Room, 3Bal)
Table 5. Result Data from Energy Performance Simulation

\begin{tabular}{ccc}
\hline $\begin{array}{c}\text { 3D Model \#1 } \\
\text { (1Bay, 2Room, 1Bal) }\end{array}$ & $\begin{array}{c}\text { Modeling } \\
\text { Settings }(1990)\end{array}$ & $\begin{array}{c}\text { Modeling } \\
\text { Setting(2016) }\end{array}$ \\
\hline & $\begin{array}{c}\text { Wall }: 0.46 \mathrm{~W} / \mathrm{m}^{2} \mathrm{~K} \\
\text { Floor }: 0.54 \mathrm{~W} / \mathrm{m}^{2} \mathrm{~K} \\
\text { Intergeneration Floor } \\
\text { (Roof) }: 0.75 \mathrm{~W} / \mathrm{m}^{2} \mathrm{~K} \\
\text { Win }: 2.85 \mathrm{~W} / \mathrm{m}^{2} \mathrm{~K}\end{array}$ & $\begin{array}{c}\text { Wall }: 0.25 \mathrm{~W} / \mathrm{m}^{2} \mathrm{~K} \\
\text { Floor }: 0.18 \mathrm{~W} / \mathrm{m}^{2} \mathrm{~K} \\
\text { Intergeneration Floor } \\
\text { Win }: 0.48 \mathrm{~W} / \mathrm{m}^{2} \mathrm{~K}\end{array}$ \\
\hline Consumption (elec) & $120 \mathrm{kWh} / \mathrm{m}^{2} / \mathrm{y}$ & $90 \mathrm{kWh} / \mathrm{m}^{2} / \mathrm{y}$ \\
\hline Consumption (fuel) & $1,172 \mathrm{MJ} / \mathrm{m}^{2} / \mathrm{y}$ & $922 \mathrm{MJ} / \mathrm{m}^{2} / \mathrm{y}$ \\
\hline Consumption (Total) & $1,606 \mathrm{MJ} / \mathrm{m}^{2} / \mathrm{y}$ & $1,248 \mathrm{MJ} / \mathrm{m}^{2} / \mathrm{y}$ \\
\hline \hline $\begin{array}{c}\text { 3D Model \#2 } \\
\text { (2Bay, 2Room, 2Bal) }\end{array}$ & $\begin{array}{c}\text { Modeling } \\
\text { Settings }(1990)\end{array}$ & $\begin{array}{c}\text { Modeling } \\
\text { Setting }(2016)\end{array}$ \\
\hline
\end{tabular}

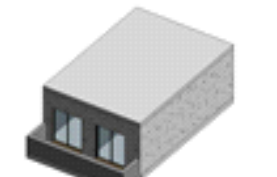

Wall : $0.46 \mathrm{~W} / \mathrm{m}^{2} \mathrm{~K}$ Floor: $0.54 \mathrm{~W} / \mathrm{m}^{2} \mathrm{~K}$ Intergeneration Floor (Roof) : $0.75 \mathrm{~W} / \mathrm{m}^{2} \mathrm{~K}$

Win : $2.85 \mathrm{~W} / \mathrm{m}^{2} \mathrm{~K}$

Wall : $0.25 \mathrm{~W} / \mathrm{m}^{2} \mathrm{~K}$ Floor : $0.18 \mathrm{~W} / \mathrm{m}^{2} \mathrm{~K}$ Intergeneration Floor (Roof) : $0.48 \mathrm{~W} / \mathrm{m}^{2} \mathrm{~K}$

Win : $1.45 \mathrm{~W} / \mathrm{m}^{2} \mathrm{~K}$

\begin{tabular}{ccc}
\hline Consumption (elec) & $105 \mathrm{kWh} / \mathrm{m}^{2} / \mathrm{y}$ & $92 \mathrm{kWh} / \mathrm{m}^{2} / \mathrm{y}$ \\
\hline Consumption (fuel) & $1,184 \mathrm{MJ} / \mathrm{m}^{2} / \mathrm{y}$ & $909 \mathrm{MJ} / \mathrm{m}^{2} / \mathrm{y}$ \\
\hline Consumption (Total) & $1,563 \mathrm{MJ} / \mathrm{m}^{2} / \mathrm{y}$ & $1,240 \mathrm{MJ} / \mathrm{m}^{2} / \mathrm{y}$ \\
\hline \hline $\begin{array}{c}\text { 3D Model \#3 } \\
\text { (2Bay, 3Room, 2Bal) }\end{array}$ & $\begin{array}{c}\text { Modeling } \\
\text { Settings(1990) }\end{array}$ & $\begin{array}{c}\text { Modeling } \\
\text { Setting }(2016)\end{array}$ \\
\hline
\end{tabular}

\begin{tabular}{|c|c|c|}
\hline & $\begin{array}{c}\text { Wall : } 0.46 \mathrm{~W} / \mathrm{m}^{2} \mathrm{~K} \\
\text { Floor : } 0.54 \mathrm{~W} / \mathrm{m}^{2} \mathrm{~K} \\
\text { Intergeneration Floor } \\
\text { (Roof) }: 0.75 \mathrm{~W} / \mathrm{m}^{2} \mathrm{~K} \\
\text { Win : } 2.85 \mathrm{~W} / \mathrm{m}^{2} \mathrm{~K}\end{array}$ & $\begin{array}{c}\text { Wall : } 0.25 \mathrm{~W} / \mathrm{m}^{2} \mathrm{~K} \\
\text { Floor : } 0.18 \mathrm{~W} / \mathrm{m}^{2} \mathrm{~K} \\
\text { Intergeneration Floor } \\
\text { (Roof) }: 0.48 \mathrm{~W} / \mathrm{m}^{2} \mathrm{~K} \\
\text { Win : } 1.45 \mathrm{~W} / \mathrm{m}^{2} \mathrm{~K}\end{array}$ \\
\hline Consumption (elec) & $106 \mathrm{kWh} / \mathrm{m}^{2} / \mathrm{y}$ & $91 \mathrm{kWh} / \mathrm{m}^{2} / \mathrm{y}$ \\
\hline Consumption (fuel) & $1,132 \mathrm{MJ} / \mathrm{m}^{2} / \mathrm{y}$ & $821 \mathrm{MJ} / \mathrm{m}^{2} / \mathrm{y}$ \\
\hline Consumption (Total) & $1,515 \mathrm{MJ} / \mathrm{m}^{2} / \mathrm{y}$ & $1,149 \mathrm{MJ} / \mathrm{m}^{2} / \mathrm{y}$ \\
\hline $\begin{array}{c}\text { 3D Model \#4 } \\
\text { (3Bay, 3Room, 2Bal) }\end{array}$ & $\begin{array}{c}\text { Modeling } \\
\text { Settings(1990) }\end{array}$ & $\begin{array}{c}\text { Modeling } \\
\text { Setting(2016) }\end{array}$ \\
\hline
\end{tabular}

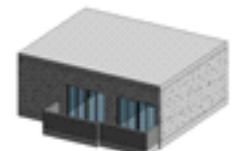

Wall : $0.46 \mathrm{~W} / \mathrm{m}^{2} \mathrm{~K}$ Floor : $0.54 \mathrm{~W} / \mathrm{m}^{2} \mathrm{~K}$

Intergeneration Floor

(Roof) : $0.75 \mathrm{~W} / \mathrm{m}^{2} \mathrm{~K}$

Win : $2.85 \mathrm{~W} / \mathrm{m}^{2} \mathrm{~K}$

Wall : $0.25 \mathrm{~W} / \mathrm{m}^{2} \mathrm{~K}$

Floor : $0.18 \mathrm{~W} / \mathrm{m}^{2} \mathrm{~K}$

Intergeneration Floo

(Roof) : $0.48 \mathrm{~W} / \mathrm{m}^{2} \mathrm{~K}$

Win : $1.45 \mathrm{~W} / \mathrm{m}^{2} \mathrm{~K}$

\begin{tabular}{ccc}
\hline Consumption (elec) & $107 \mathrm{kWh} / \mathrm{m}^{2} / \mathrm{y}$ & $97 \mathrm{kWh} / \mathrm{m}^{2} / \mathrm{y}$ \\
\hline Consumption (fuel) & $1,070 \mathrm{MJ} / \mathrm{m}^{2} / \mathrm{y}$ & $782 \mathrm{MJ} / \mathrm{m}^{2} / \mathrm{y}$ \\
\hline Consumption (Total) & $1,454 \mathrm{MJ} / \mathrm{m}^{2} / \mathrm{y}$ & $1,131 \mathrm{MJ} / \mathrm{m}^{2} / \mathrm{y}$ \\
\hline \hline $\begin{array}{c}\text { 3D Model \#5 } \\
\text { (3Bay, 3Room, 3Bal) }\end{array}$ & $\begin{array}{c}\text { Modeling } \\
\text { Settings(1990) }\end{array}$ & $\begin{array}{c}\text { Modeling } \\
\text { Setting }(2016)\end{array}$ \\
\hline
\end{tabular}

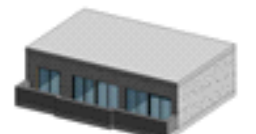

Wall : $0.46 \mathrm{~W} / \mathrm{m}^{2} \mathrm{~K}$

Floor : $0.54 \mathrm{~W} / \mathrm{m}^{2} \mathrm{~K}$

Intergeneration Floor

(Roof) : $0.75 \mathrm{~W} / \mathrm{m}^{2} \mathrm{~K}$

Wall : $0.25 \mathrm{~W} / \mathrm{m}^{2} \mathrm{~K}$

Win : $2.85 \mathrm{~W} / \mathrm{m}^{2} \mathrm{~K}$

Floor : $0.18 \mathrm{~W} / \mathrm{m}^{2} \mathrm{~K}$

Intergeneration Floo

(Roof) : $0.48 \mathrm{~W} / \mathrm{m}^{2} \mathrm{~K}$

Win : $1.45 \mathrm{~W} / \mathrm{m}^{2} \mathrm{~K}$

\begin{tabular}{lll}
\hline Consumption (elec) & $100 \mathrm{kWh} / \mathrm{m}^{2} / \mathrm{y}$ & $91 \mathrm{kWh} / \mathrm{m}^{2} / \mathrm{y}$ \\
\hline Consumption (fuel) & $1,003 \mathrm{MJ} / \mathrm{m}^{2} / \mathrm{y}$ & $731 \mathrm{MJ} / \mathrm{m}^{2} / \mathrm{y}$ \\
\hline Consumption (Total) & $1,362 \mathrm{MJ} / \mathrm{m}^{2} / \mathrm{y}$ & $1,059 \mathrm{MJ} / \mathrm{m}^{2} / \mathrm{y}$ \\
\hline
\end{tabular}

Fig. 2. Modeling of Five Units Using the Revit Modeling Tool 
Table 6-1. Result Data from Daylighting Simulation

\begin{tabular}{|c|c|c|c|}
\hline $\begin{array}{c}\text { No } \\
\text { Balcony }\end{array}$ & $\begin{array}{c}\text { Daylight } \\
\text { View } 1 \\
\end{array}$ & $\begin{array}{c}\text { Daylight } \\
\text { View } 2 \\
\end{array}$ & $\begin{array}{l}\text { Daylight } \\
\text { View } 3 \\
\end{array}$ \\
\hline $\begin{array}{l}\text { Winter } \\
\text { Solstice } \\
10 \mathrm{AM}\end{array}$ & & & - \\
\hline $\begin{array}{l}\text { Winter } \\
\text { Solstice } \\
\text { 4PM }\end{array}$ & & & - \\
\hline Balcony & $\begin{array}{c}\text { Daylight } \\
\text { View } 1\end{array}$ & $\begin{array}{c}\text { Daylight } \\
\text { View } 2\end{array}$ & $\begin{array}{c}\text { Daylight } \\
\text { View } 3\end{array}$ \\
\hline $\begin{array}{l}\text { Winter } \\
\text { Solstice } \\
\text { 10AM }\end{array}$ & & & - \\
\hline $\begin{array}{l}\text { Winter } \\
\text { Solstice } \\
\text { 4PM }\end{array}$ & & & - \\
\hline $\begin{array}{c}\text { Mullion } \\
\left(0^{\circ}\right)\end{array}$ & $\begin{array}{c}\text { Daylight } \\
\text { View } 1\end{array}$ & $\begin{array}{c}\text { Daylight } \\
\text { View } 2\end{array}$ & $\begin{array}{c}\text { Daylight } \\
\text { View } 3\end{array}$ \\
\hline $\begin{array}{l}\text { Winter } \\
\text { Solstice } \\
\text { 10AM }\end{array}$ & & & \\
\hline $\begin{array}{l}\text { Winter } \\
\text { Solstice } \\
\text { 4PM }\end{array}$ & & & \\
\hline $\begin{array}{l}\text { Mullion } \\
\left(15^{\circ}\right)\end{array}$ & $\begin{array}{c}\text { Daylight } \\
\text { View } 1\end{array}$ & $\begin{array}{c}\text { Daylight } \\
\text { View } 2\end{array}$ & $\begin{array}{c}\text { Daylight } \\
\text { View } 3\end{array}$ \\
\hline $\begin{array}{c}\text { Winter } \\
\text { Solstice } \\
\text { 10AM }\end{array}$ & & & \\
\hline $\begin{array}{l}\text { Winter } \\
\text { Solstice } \\
\text { 4PM }\end{array}$ & & & \\
\hline $\begin{array}{c}\text { Mullion } \\
\left(30^{\circ}\right)\end{array}$ & $\begin{array}{l}\text { Daylight } \\
\text { View } 1\end{array}$ & $\begin{array}{l}\text { Daylight } \\
\text { View } 2\end{array}$ & $\begin{array}{c}\text { Daylight } \\
\text { View } 3\end{array}$ \\
\hline $\begin{array}{l}\text { Winter } \\
\text { Solstice } \\
\text { 10AM }\end{array}$ & & & \\
\hline $\begin{array}{l}\text { Winter } \\
\text { Solstice } \\
\text { 4PM }\end{array}$ & & & \\
\hline
\end{tabular}

Table 6-2. Result Data from Daylighting Simulation

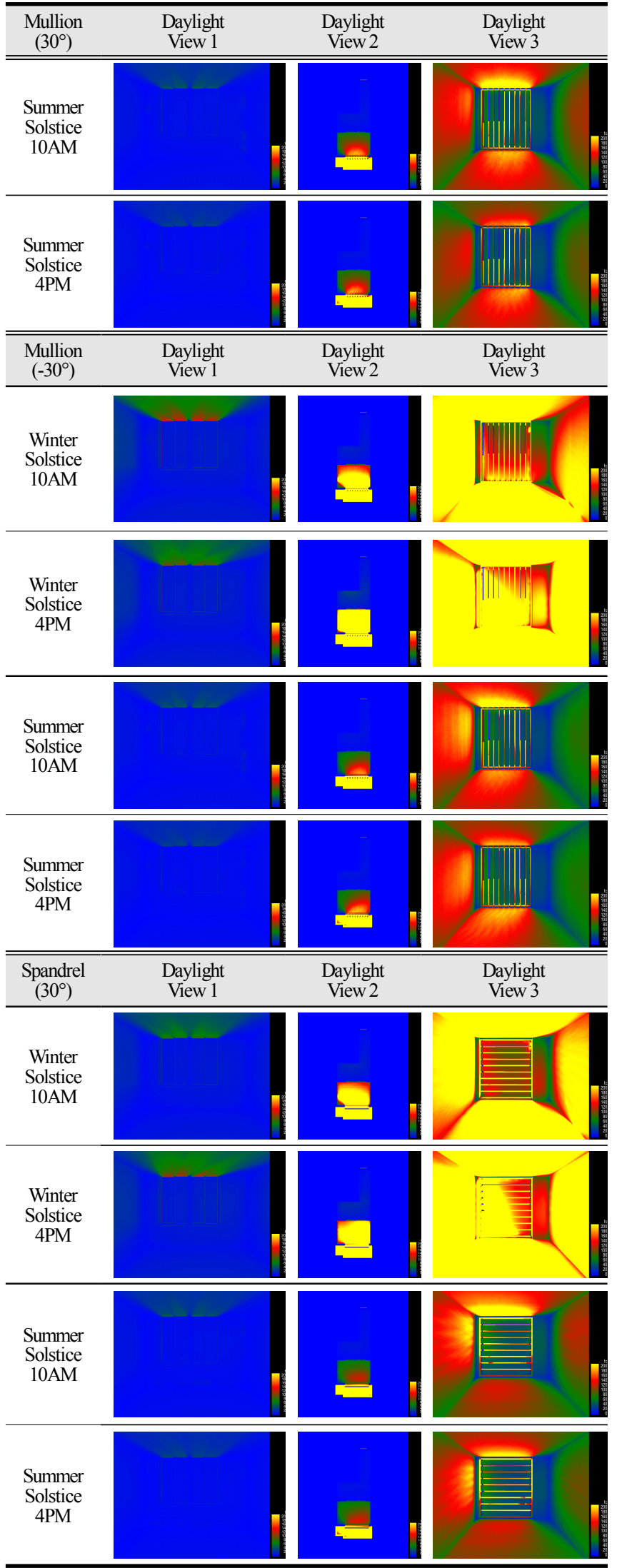

This research also simulated about the changes daylighting by the changes louver forms and window forms. The above table 
shows result data, and this daylighting simulation was simulated 40-59 housing unit(3D Model \#1). Then, the unit was changed in variety forms as a type of no balcony, with balcony, with mullion louver $0^{\circ}$, with mullion louver $15^{\circ}$, with mullion louver $30^{\circ}$, with mullion louver $-30^{\circ}$ and with spandrel louver $30^{\circ}$.

Also, Daylight View 1 and 3 are perspective view and Daylight View 2 is plan view. Simulation time is totally two types, one of time type is winter solstice and the other is summer solstice. Each time type has two measurement time points as 10AM and 4PM.

In sum, the above simulations have suggested findings as follows; electricity is saved for 4,330.7 $\mathrm{kWh}$ per year, fuel is saved for $17,160 \mathrm{kWh}$ per year, and the total energy can be saved with $21,490 \mathrm{kWh}$ a year.

\section{CONCLUSION}

This research reclassified and reorganized applicable factors into the existing apartment houses due to some limitations of the existing energy-saving system such as low applicability with the existing houses and high-rise buildings. And this study also analyzed types of apartment houses in the 1990s-2000s that need to be remodeled immediately. For this process, 3D models were constructed and a series of simulations were performed for analyses on energy performance and energy effectiveness of each change on thermal standard and heat insulation property of wall and floor.

This research then analyzed the change of electricity and fuel consumption according to the change of insulation standard. As results of the above simulations, the following findings have been confirmed; electricity is saved for $4,330.7 \mathrm{kWh}$ per year, fuel is saved for $17,160 \mathrm{kWh}$ per year, and the total energy can be saved with $21,490 \mathrm{kWh}$ a year. Therefore, it is expected that energy savings will also increase in case the area is increased because simulation unit is normally smaller than house unit.

And results of daylight simulation is almost test model have had overmany daylight. So, this research analyzed about method to decrease the daylight. because over daylight may be cause over cooling load in summer. And then, result of simulation is that installing an angle louver can be decrease the daylight. Also in winter, mullion louver and spandrel louver seem almost similar efficiency. but in summer, spandrel louver is more efficiency than mullion louver.

Finally, this research is expected to serve as fundamental findings and new trials for energy housing systems and suggest new methods applicable to the existing housings and buildings. This research has a limitation that the suggested data have not been proven by experimental procedures for real or scaled spaces, and this part remains as an ongoing study.

\section{Acknowledgements}

This research was developed from Master's Thesis by Mu-Jin Jo joined as an industrial collaborator and supported by a grant from Land and Transport Technology Promotion Research Program (Implementation of Building Façade Modules Based on Colorful Semi-transparent Solar Cells towards Urban Surroundings) funded by Ministry of Land and Transport Affairs of Korean Government (Project No.: 17CTAP-C129910-01).

\section{Reference}

[1] Kim, Dae-Sik, "Plan for Apartment House Remodeling - Focused on the city and housing view improvement, and eco-friendly remodeling-, Hong-Ik University, 2009

[2] Choi, Woo-Young, Lee, Da-Hye, Park, Yong-Ho, Lee, Sang-ho, "A Case Study on Diagnostic Simulation Method of Energy Efficiency for The Remodeling of Building", Journal of the Architectural Institute of Korea, Vol.24, No.2, 2004, pp.183-186

[3] Lee, Kyu-Yong, Seo, Jang-Hoo, Kim, Yong-Seong, "A Preliminary Study on Green IT Based Zero Energy House Through an Example of Domestic Experimental Housing", Journal of Korea Digital Architecture and Interior, Vol.14, No.1, 2014

[4] Kim, Sun-Geun, Kwon, Soon-Wook, "The Study of Optimum Method About the Design and Operating Through a Zero-Energy House Built in the Existing Building", Journal of The Korean Society For New And Renewable Energy, Vol.11, No.1, 2015, pp.49-59

[5] Seo, Ji-Won, Lim, Jae-Han, Song, Seung-Yeong, "Case Study of Energy-Saving Effect Through Renovation of Old Apartment Building", Journal of Korea Institute of Architectural Sustainable Environment And Building Systems, 2011

[6] Shin, Hyun-Cheol, Jang, Gun-Eik, "Analysis of Energy Consumption and Cost based on Combination of Element Technologies for Implementing Zero-Energy House", Journal of Korea Institute of Architectural Sustainable Environment And Building Systems, Vol.9, No.2, 2015, pp.163-170

[7] Lee, Chung-Kook, Lee, Jeong-Cheol, Kim, Sang-Su, Suh, Seung-Jik, "Suggestion of the Characteristics of Element Technology and the Standard Model through the Comparison of Domestic Zero-Energy Houses", Journal of Korea Society of Geothermal Energy Engineers, Vol.8, No.2, 2012, pp.27-35

[8] Im, Jeong-Ah, "Environmental Performance Evaluation for the Remodeling of Apartment Housing at the Planning Stage", Chung-Buk University, 2008

[9] Yoo, Jae-Rin, "A Study on Remodeling Evaluation of Aged Apartment housing by Energy Analysis", In-Cheon University, 2003

[10] Ministry of Land and Infrastructure, "Enalbe Research Plan of Green Remodeling Target to Old Houses, 2013

[11] Seoul, "2025 Remodeling Plan of Seoul Apartment House", 2016

[12] Jo, Mu-Jin, Han, Seung-Hoon, "A Standard Remodeling System for Existing Housing Units Integrated with The Energy Production Module Using Algae Activation", Proceedings of 30th Research World International Conference, 2017

[13] Jo, Mu-Jin, "A Study on the Remodeling System of Energy Performance Improvements Applicable to Existing Housing Unit", Master's Thesis, Chonnam National University, 2017 